\title{
A Scalable Petri Net Representation of Interaction Protocols for Overhearing*
}

\author{
Gery Gutnik and Gal Kaminka \\ The MAVERICK Group \\ Computer Science Department, Bar-Ilan University, Israel \\ \{gutnikg@cs.biu.ac.il,galk@cs.biu.ac.il\}
}

\begin{abstract}
Representing agent conversations is an important aspect of multi-agent applications. Lately, Petri nets have been found to provide certain advantages comparing to other representation approaches. Radically different approaches using Petri nets have been proposed, and yet their relative strengths and weaknesses have not been examined. This paper analyzes the existing Petri net representations in terms of their scalability and appropriateness for monitoring tasks. Based on the insights gained, we propose a scalable Petri net representation for overhearing.
\end{abstract}

\section{Introduction}

Representing agent conversations is an important aspect of open distributed multi-agent systems. Lately, Petri nets have been shown to offer significant advantages in representing multi-agent interactions $[1,5,6]$. Different approaches using Petri nets have been introduced, and yet their relative strengths and weakness have not been investigated.

This paper addresses this open challenge. First, we present a basic classification of existing approaches (Section 2). Then, these approaches are analyzed and compared (Section 3). Based on the insights gained, a scalable Petri net representation for overhearing is proposed (Section 4). Finally, Section 5 concludes.

\section{Related Work Classification}

In general, existing Petri net approaches for modeling multi-agent interactions use one of the two following design choices.

Individual roles and Colored Petri nets (CP-nets). Most investigations choose to separately represent individual roles within the interaction $[1,2,4,5]$. Separate places are used for separate roles, and thus different markings distinguish a conversation state where one agent has sent a message, from a state where the other agent received it. All these investigations use CP-nets.

\footnotetext{
* This research was supported in part by BSF grant \#2002401
}

Joint-states and Place/Transition nets (PT-nets). In contrast, a limited number of investigations use PTnets with joint conversation states $[6,7]$. In joint state representations, each net place is at once a representative of the conversation state of all agents. Typically, markings represent only valid conversation states.

\section{Analysis of Key Representations}

This section presents a comparative analysis of existing approaches on the basis of scalability (Section 3.1), and suitability for monitoring tasks (Section 3.2).

\subsection{Scalability}

In principle, for a conversation that has $R$ roles, with $M$ messages, a representation which explicitly differentiates the conversation state of each role would have $\mathrm{O}(M R)$ places: for every message there would be two individual places for the sender (before sending, and after sending), and similarly two more for each receiver. All possible joint states (i.e. message sent and received, sent and not received, etc) can be represented.

However, many applications only require representation of valid conversation states (message not sent and not received, or sent and received). Under such assumption, for every message, there are only two joint states regardless of the number of roles: before the message is sent, and after the message is sent and received. Thus, the number of places in joint-state representation is $\mathrm{O}(M)$.

We now turn to examining the use of color tokens. In principle, CP-nets and PT-nets are equivalent from a computational perspective. However, when representing conversations, a significant difference between PT-nets and CP-nets is their scalability. A PT-token corresponds to a single bit. The information it conveys is a function of the place it is marking. As a result, it is impossible to represent several concurrent conversations on the same PT-net. Thus, representing $C$ concurrent conversationsof the same interaction protocol-requires $\mathrm{O}(C)$ PT-nets.

In contrast, colored tokens can be differentiated, even when multiple tokens mark the same net. Thus, it allows 
us to represent multiple concurrent conversations-of the same protocol-on a single CP-net structure.

\begin{tabular}{|c|c|c|}
\hline & PT-nets & CP-nets \\
\hline $\begin{array}{c}\text { Individual } \\
\text { States }\end{array}$ & $\begin{array}{c}\text { Space: } \\
\text { O(MRC) }\end{array}$ & $\begin{array}{c}\text { Space: O(MR) } \\
{[1],[2],[4],[5]}\end{array}$ \\
\hline $\begin{array}{c}\text { Joint } \\
\text { States }\end{array}$ & $\begin{array}{c}\text { Space: } \mathbf{O}(\mathbf{M C}) \\
{[6],[7]}\end{array}$ & Space: O(M) \\
\hline
\end{tabular}

Table 1. Scalability comparison.

Table 1 summarizes the space complexity of different approaches, given that we model $C$ conversations, each with a maximum of $R$ roles, and $M$ messages.

\subsection{Monitoring Conversations}

Focusing on monitoring, we distinguish two settings, depending on the information available to the monitor.

In the first settings, the monitor, representing the conversation, has access to the state of the conversation in one or more of the participants, but not to the messages being exchanged. Thus, messages are not explicitly represented, except as transitions that take the conversation from one place to another. By placing tokens in conversation places, an agents' state can be inferred. Then, letting the corresponding transition fire implies the message being sent and received. Previous investigations using this approach include $[1,4,5]$.

In the second settings, the monitor has knowledge of the messages being sent and received, but does not necessarily know the internal conversation state. It monitors conversations by tracking the messages (e.g., through overhearing). This requires the use of separate message places. In this type of representation, a state place and a message place are connected via a transition to a new state. A monitoring agent places a token in the appropriate message place whenever it intercepts a message. Together with conversation state places, these tokens allow the conversation to transition from one conversation state to another only based on explicit knowledge of the message being sent or received. Here, previous investigations include [1,2,6,7].

\section{Scalable Representation for Overhearing}

Based on the insights gained, we propose a novel scalable Petri net representation for overhearing. In this approach, multi-agent interactions are modeled using CP-nets where places explicitly denote joint conversation states and messages. Figure 1 below shows an example of our representation approach.

This representation can be used to cover essentially all features used in FIPA conversation standards. Further details can be found in [3].

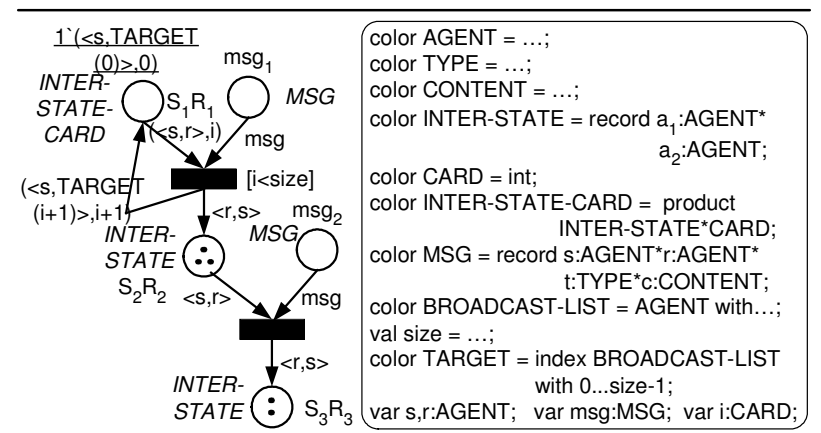

Figure 1. Broadcast in CP-net representation.

\section{Conclusion}

Recently, increasing attention has been directed at representing multi-agent conversations using Petri nets. Unfortunately, features of competing approaches with respect to scalability and suitability for different tasks have not been analyzed.

This paper sought to address this open challenge. We analyzed key features of existing representations showing that (i) when representing valid conversations, a CP-net, where places denote joint conversation states, scales better than other approaches; (ii) message places are necessary for tracking conversations by overhearing. Unfortunately, previous work did not examine this combination. We propose to use this representation, $\mathrm{CP}$ nets with joint states and message places, to target scalable overhearing and monitoring tasks.

\section{References}

[1] Cost, R. S., Chen, Y., Finin, T., Labrou, Y. \& Peng, Y. (2000). Using Coloured Petri Nets for a Conversation Modeling. In Dignum, F. \& Greaves, M. (Eds.), Issues in Agent Communications, pp. 178-192. Springer-Verlag.

[2] Cranefield S., Purvis M., Nowostawski M. \& Hwang P. (2002). Ontologies for interaction protocols. In Proceedings of AAMAS-02.

[3] Gutnik, G. \& Kaminka, G.A. (2004). A comprehensive Petri net representation for multi-agent conversations. MAVERICK Technical Report 2004/1, Bar-Ilan University, at www.cs.biu.ac.il/ maverick/tech-reports/.

[4] Lin, F., Norrie, D. H., Shen, W. \& Kremer, R. (2000). A schema-based approach to specifying conversation policies. In Dignum, F. \& Greaves, M. (Eds.), Issues in Agent Communications, pp. 193-204. Springer-Verlag.

[5] Mazouzi, H., Fallah-Seghrouchni, A. E. \& Haddad, S. (2002). Open protocol design for complex interactions in multi-agent systems. In Proceedings of AAMAS-02.

[6] Nowostawski, M., Purvis, M. \& Cranefield, S. (2001). A layered approach for modeling agent conversations. In Proceedings of Workshop on Infrastructure for Agents, MAS and Scalable MAS, pp. 163-170. Montreal, Canada.

[7] Poutakidis, D., Padgham, L. \& Winikoff, M. (2002). Debugging multi-agent systems using design artifacts. In Proceedings of AAMAS-02. 